\title{
Silicon Simulation Code for Belle II and ILC
}

\section{Zbynek DRASAL *i}

Charles University Prague, Czech Republic

E-mail: drasaleipnp.troja.mff.cuni.cz

\section{Kolja PROTHMANN}

MPI München, Germany

E-mail: kolja@mpp.mpg.de

\section{Benjamin SCHWENKER ${ }^{\S}$}

Georg-August-Universität Göttingen, Germany

E-mail: benjamin.schwenker@phys.uni-goettingen.de

Monte Carlo simulations in high-energy physics experiments face the non-trivial task of simulating realistically the response of individual detector components, while keeping the costs in terms of CPU time reasonably low. Such simulation procedures are called digitization and have to incorporate detector physics in as much detail as possible, while performing fast enough. Here, we present our approach to the simulation of the Belle II vertex detector (VXD) using the ILC software framework. We simulate the response of DEPFET (DEPleted Field Effect Transistor) pixel detectors (PXD) and double-sided silicon micro-strip detectors (SVD) in the presence of a magnetic field. To achieve sufficient performance, we use a combination of fast numerical techniques and reasonable simplification of in-detector physics. The simulation itself is divided into 3 steps: particle propagation through matter (using Geant 4 with a detailed implementation of the VXD geometry), charge collection in the silicon detectors (digitization) and clustering. The second and third steps are performed in separate reconstruction modules - Marlin modules: SiPxlDigi (pixel detectors) and SiStripDigi (micro-strip detectors). The following physics processes are considered: continuous energy loss fluctuations, generation of e-h pairs, drift in electric field, diffusion, Lorentz shift, mutual crosstalk (strips), read-out/geometric pitch effect (strips), electronics/digital noise. The clustering procedure is based on the center-of-gravity and analog head-tail algorithms. All effects have been studied and compared to test beam data in order to validate the algorithms and to determine the relative importance of individual processes.

The 20th Anniversary International Workshop on Vertex Detectors - VERTEX 2011

June 19 - 24, 2011

Rust, Lake Neusiedl, Austria

\section{* Speaker.}

${ }^{\dagger}$ This work has been supported by the Czech Science Foundation Grant No. 203/10/0777 and the Ministry of Education, Youth and Sports of the Czech Republic No. LA10033 and MSM0021620859.

† This work has been supported by the DFG cluster of excellence "Origin and Structure of the Universe" of Germany.

$\S$ This work has been supported by the Volkswagen Foundation. 


\section{Introduction}

SuperKEKB, an upgrade of the successful asymmetric $\mathrm{e}^{+} \mathrm{e}^{-}$collider and current world luminosity record holder $\left(2.11 \times 10^{34} \mathrm{~cm}^{-2} \mathrm{~s}^{-1}\right)$ KEKB (Tsukuba, Japan), is foreseen by fall 2014 [1]. The main goal of the new Super Flavour Factory is to dramatically increase statistics by a factor of 40 and thus deliver an integrated luminosity of $50 \mathrm{ab}^{-1}$ by end of 2021 . For this reason, the nanobeam option has been chosen, which, as a side effect, leads to a significant increase in background level, with Touschek effect being a dominant component. To exploit the new conditions and provide high-precision measurements of the decay vertex of B meson systems, a new Belle II silicon vertex detector will be operated. Due to the increased background level and required sensitivity to low momenta particles, a low material budget pixel detector closest to the interaction point is a must. Belle II VXD will consist of 2 layers of $75 \mu \mathrm{m}$ thick DEPFET-type pixel detectors and 4 barrel layers of $320 \mu \mathrm{m}$ thick double-sided strip detectors, complemented with 3 forward layers. Precise and fast silicon ( $\mathrm{Si}$ ) simulations are absolutely necessary to optimize such a detector.

\section{Software Framework}

Due to its convenient modular design (Fig. 1) and sufficient flexibility, a software framework for ILC (International Linear Collider) [2] has been adopted to the special needs of Belle II experiment and new software packages: SiPxlDigi and SiStripDigi have been developed. The framework

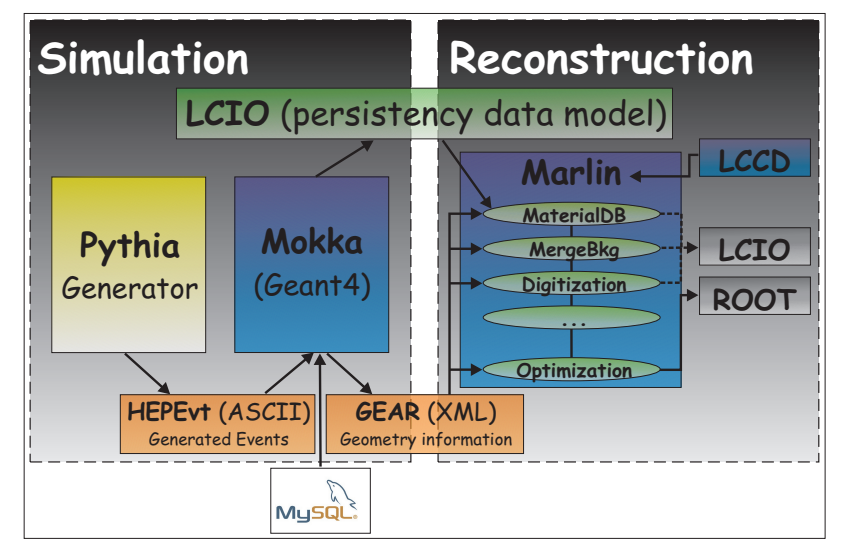

Figure 1: Scheme of ILC software framework

itself provides a typical chain of high energy physics tools: generator (Pythia), simulation (Mokka - Geant4 based toolkit), digitization (Marlin modules: SiPxlDigi \& SiStripDigi), reconstruction (Marlin module: SiTracking and other modules) and analysis. As the geometry information is required with different level of detail throughout the chain, two different interfaces are provided. An interface to access a MySQL database at Geant4 level (with full detail of detector geometry) and a GEAR interface at reconstruction level (with limited geometry description provided via XML). The data model, which is used to describe the event data throughout the chain, is based on the LCIO persistency framework. It provides all necessary structures ( ++ objects) to store data produced in silicon simulation: simulated hits (SimTrackerHits) $\rightarrow$ digits (TrackerPulses) $\rightarrow$ hits (TrackerHits), etc. All data types hold the relation to MC truth information (MCParticle). 


\section{Silicon Simulation \& Geant4 Toolkit}

Several specific issues closely related to Geant 4 will be addressed in this section. The first issue is the right choice of a suitable physics model describing continuous energy loss fluctuations for ionizing particles traversing a silicon detector. Years ago, when Geant3 (Fortran-based predecessor of Geant4) was used, we noticed that the default model (denoted as G3 Landau) didn't provide correct distributions of $\mathrm{dE} / \mathrm{dx}$ for thin material (typically 10-100 $\mu \mathrm{m}$ of Si absorber). The main limitation is that, in the Landau model, the typical energy loss in the absorber should be large compared to the binding energy of the most tightly bound electron, which was not fullfilled in thin silicon sheets. The more accurate Photo-Absorption Ionisation (PAI) model, which takes into account the atomic structure of the absorber, provided accurate description of measured data, but with more CPU needed. For details see [3].

Similarly, Geant 4 comes with several different models. The G4UniversalFluctuations model is used by default. It is based on a simplified model of atoms with two energy levels, and an atom-particle interaction that can either be an excitation, with energy loss $E_{1}$ or $E_{2}$, or ionisation, distributed according to $1 / E^{2}$ distribution [ [ enough, one can use the G4PAIModel instead. In order to validate the physics model, two detailed simulations in Geant 3 and Geant4, with $8 \mathrm{GeV} / \mathrm{c}$ pions traversing $290 \mu \mathrm{m}$ thick silicon bulk have been performed and compared to real data cited in [5]. The results are shown in Fig. 2 and summarized in Table 1. The discrepancy between the Geant 4 simulation (using either two-levels atom model or PAI model) and the experimental data is reasonably small.

\begin{tabular}{lccccc}
\hline & G3 Landau & G3 PAI & G4 Universal & G4 PAI & Experiment \\
\hline MPV $\left\langle\frac{d E}{d x}\right\rangle[\mathrm{keV}]$ & $96 \pm 1$ & $79 \pm 1$ & $84 \pm 1$ & $81 \pm 1$ & 79.43 \\
FWHM $w[\mathrm{keV}]$ & $20 \pm 1$ & $29 \pm 1$ & $30 \pm 1$ & $30 \pm 1$ & 29.24 \\
\hline
\end{tabular}

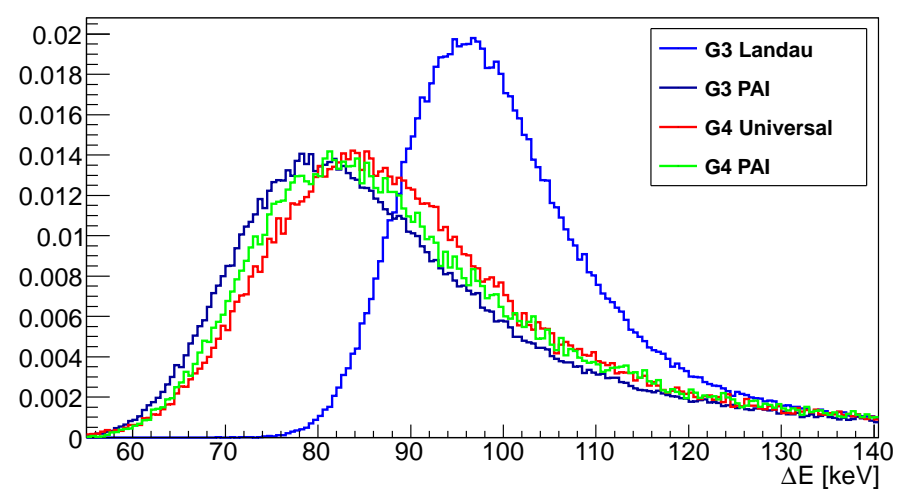

Figure 2: and Table 1: Energy loss distributions of $8 \mathrm{GeV} / \mathrm{c}$ pions in $290 \mu \mathrm{m}$ thick silicon for different Geant 3 and Geant 4 models. MPV stands for the most probable value and depends on material thickness, etc.

Another issue is the correct setting of a Geant4 production threshold cut, which has strong impact on the number of generated $\delta$-electrons. The optimal choice of the cut defines a compromise between the spatial precision of the simulation and the total number of generated particles. Generally speaking, if the kinetic energy of a secondary electron is below the threshold cut, no soft 
secondary particle is generated and the transferred energy is simulated as a continuous energy loss by the incident particle. There are two use cases: in case of a test beam (TB) study, one wants to precisely describe measured data and avoid systematic shift in the reconstruction of a cluster position due to incident $\delta$-electron. Such a shift might be comparable to detector precision, i.e. several $\mu \mathrm{m}$. As a solution, we recommend to set the cut so that $T_{\text {cut }} \ll M P V$, which automatically generates a realistic number of $\delta$-electrons. In a full simulation, on the other hand, the main concern is speed, which is related to the number of simulated particles. In this situation, the preferrable choice is $T_{\text {cut }} \gg M P V$. In order to demonstrate that the amount of $\delta$-electrons is far from being negligible and to study how they contribute to the energy distribution tail, we have simulated energy losses in $75 \mu \mathrm{m}$ thick silicon and set $T_{\text {cut }} \ll M P V$ (see Fig. 3).

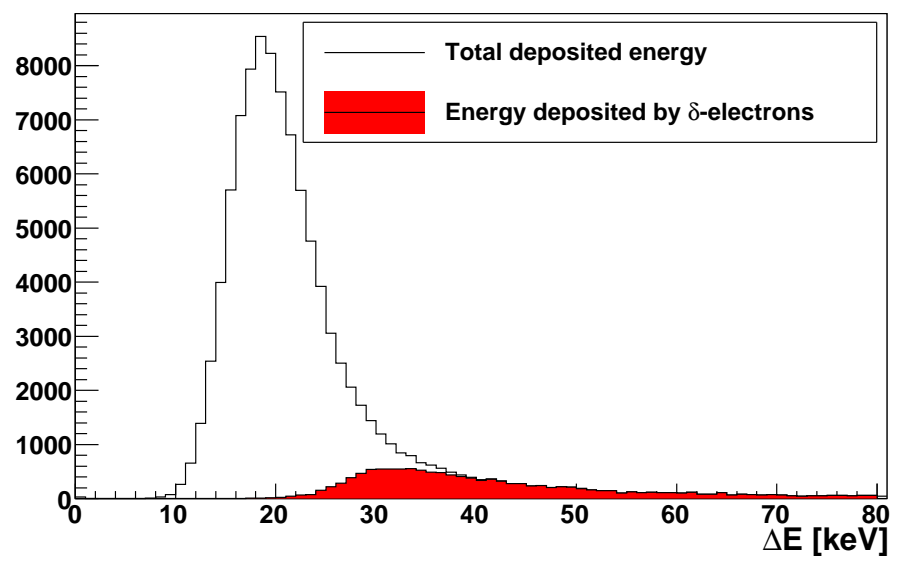

Figure 3: Deposited energy by $0.5 \mathrm{GeV} / \mathrm{c}$ pions in $75 \mu \mathrm{m}$ thick silicon (threshold cut set to $0.4 \mu \mathrm{m}$ ). Red histogram corresponds to the fraction of deposited energy generated by $\delta$-electrons.

The last issue concerns the definition of a sensitive detector and Geant4 step size calculation. In a full simulation, we use the default Geant 4 optimal calculation of individual steps (2-3 steps per detector) and accumulate them to create 1 SimTrackerHit per particle and detector (except for loopers). The main advantage of such procedure is its simplicity (1 MC particle $\leftrightarrow 1$ SimTrackerHit) and speed. The disadvantage is that fluctuations are not simulated precisely and have to be re-sampled at digitization level again. In case of detector resolution studies (no speed issues) we force Geant 4 to set the step size comparable to detector spatial resolution and thus simulate the fluctuations precisely. For comparison of different approaches see Fig. 7 .

\section{Digitization}

Digitization is the simulation of detector response to ionizing particles and/or photons in magnetic field, based on detector physics. We implemented the digitization in two new Marlin packages: SiPxlDigi, simulating the response of a DEPFET pixel detector, and SiStripDigi, simulating the response of either single- or double-sided micro-strip detector. We would note that the implementation is very generic and can be easily adapted to various pixel and/or micro-strip technologies. 


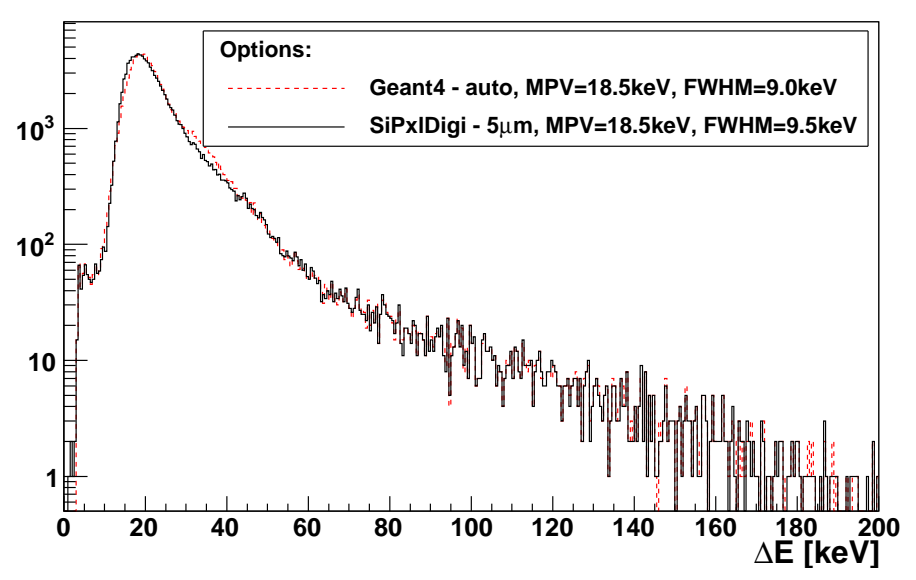

Figure 4: Comparison of energy loss fluctuations by $0.5 \mathrm{GeV} / \mathrm{c}$ pions in $80 \mu \mathrm{m}$ thick silicon generated at Geant4 level, with MPV $=18.5 \mathrm{keV}$ and FWHM $=9.0 \mathrm{keV}$ (red dashed line), versus digitization level, with $\mathrm{MPV}=18.5 \mathrm{keV}$ and FWHM $=9.5 \mathrm{keV}$ (black solid line). To emphasize agreement in distribution tails, logscale is used.

\subsection{Geometry and Data Flow}

At the input, the packages require SimTrackerHits collected within a detector integration time window. At the output, the TrackerPulses or TrackerHits (after clustering) are saved. All hits have relations to MC truth information and if more than one particle has contributed to the signal creation, the one with the highest weight is saved. The typical vertex geometry design consists of several silicon layers, each consisting of a few ladders and each ladder has usually more than one active sensor (right Fig. . $)$. In order to separate the physics model and geometry implementation, each package has its own geometry interface providing all necessary information. The whole digitization procedure is performed within the sensor local coordinate system, where for VXD it is defined as follows: $x^{\prime}$-axis is perpendicular to the sensor plane and to the beam axis, $y^{\prime}$-axis lies in a sensor plane and is perpendicular to the beam axis, $z^{\prime}$-axis is parallel to the beam axis. Position $(0,0,0)$ is defined such as the $x^{\prime}, y^{\prime}, z^{\prime}$ coordinates are always positive (right Fig. 5).
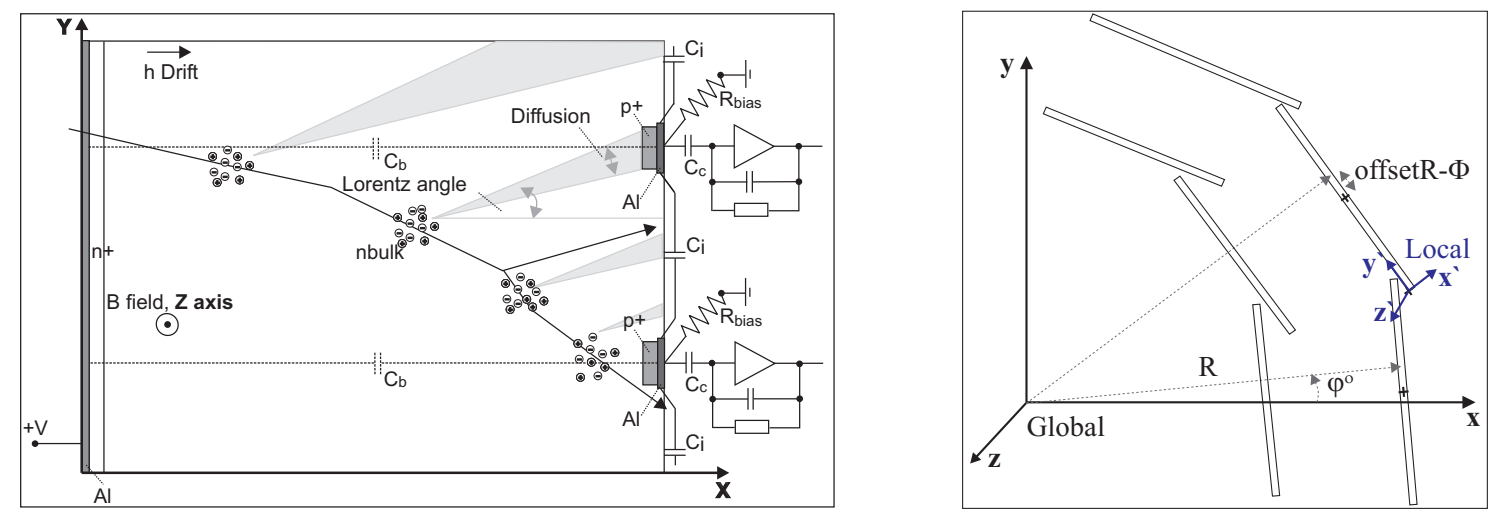

Figure 5: Left: Schematic layout of charge collection in silicon micro-strip detector. Right: Local $\overrightarrow{x^{\prime}}$ (in blue) versus global $\vec{x}$ (in black) reference system with detailed VXD layer $\rightarrow$ ladder structure. 


\subsection{SiStripDigi - Physics Model}

After a Geant4 particle enters a sensitive detector, bunches of e-h pairs are equidistantly generated along its path, with a user-defined segment of several $\mu \mathrm{m}$ (see left Fig. 5). The segment size is usually set at the level of the detector intrinsic resolution $\approx 5-20 \mu \mathrm{m}$. A fraction of charge, which is then assigned to each bunch of e-h pairs, is either calculated as a ratio of the total deposited charge, associated with each simulated hit, divided by the number of segments, or simulated through internal implementation of Geant4 G4UniversalFluctuation routine. The first method is preferred if Geant 4 step size can be set to the required precision (TB studies). The latter approach is used when no limitation to Geant 4 step size has been applied and one wants to take into account the energy loss fluctuations with the required spatial accuracy. As the fluctuation depends on particle type and its energy, detailed calculations of mean ionisation losses are required by the G4UniversalFluctuation and thus different Geant 4 models have been utilized: G4BetheBlochModel for hadrons, G4MuBetheBlochModel for muons and G4Mol lerBhabhaModel for electrons \& positrons [4]. The disadvantage of that approach is that it may require a crosscheck with Geant 4 (see Fig. $\bigoplus$ ). Finally, the number of created e-h pairs in each bunch is calculated and Poisson fluctuated. The energy needed to create an e-h pair is $3.65 \mathrm{eV}$.

All generated electrons (holes) are drifted in the electric field towards $n$-type ( $p$-type) electrodes. As micro-strip sensors have a non-trivial distribution of the electric field and thus the calculation of the weighting field would be necessary (see [3]), we have simplified the micro-strip structure to a simple p-n junction. Importantly, we have retained the dependence of carrier mobility on temperature and carrier position $x$. The dependence is defined in (4.1) and $v_{\mathrm{m}}, E_{\mathrm{c}}, \beta$ are temperature dependent parameters [6]. The strong dependence of mobility on temperature and corresponding intensity is illustrated in Fig.6.
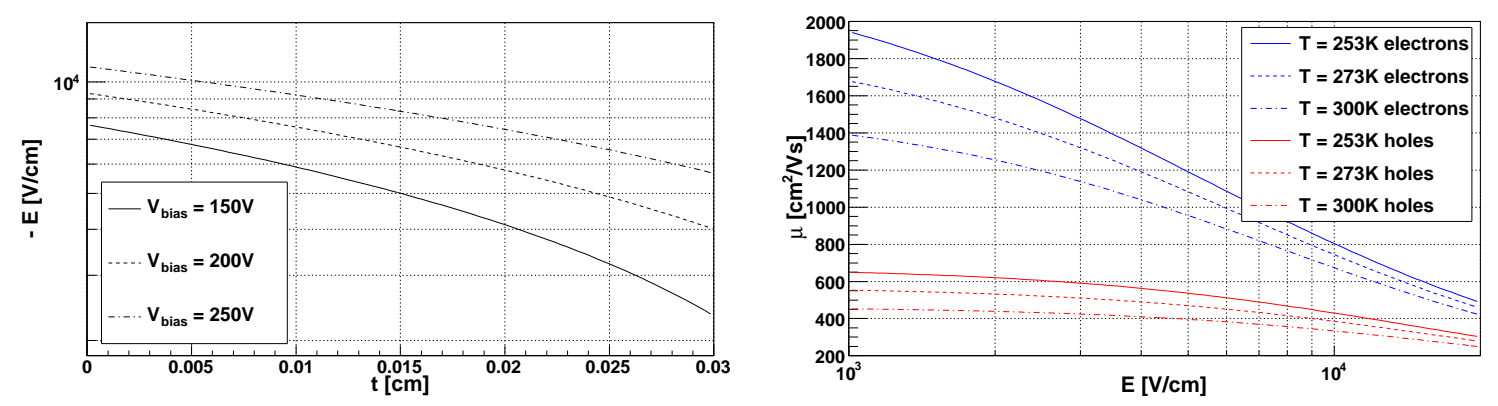

Figure 6: Left: Electric intensity versus position in a $300 \mu \mathrm{m}$ thick silicon sensor for different bias voltages: 150 (solid), 200 (dashed) and $250 \mathrm{~V}$ (semi-dashed). Right: Corresponding mobility of electrons (blue) and holes (red) for different temperature: 253 (solid), 273 (dashed) and $300 \mathrm{~K}$ (semi-dashed).

Solving the following ordinary differential equation (4.1):

$$
v(x(t))=\mu E(x) \quad\left\{\begin{array}{l}
E(x)=-\left(\frac{V+V_{\text {dep }}}{d}-\frac{2 x V_{\text {dep }}}{d^{2}}\right) \\
\mu(E(x), T)=\left(\frac{v_{\mathrm{m}} / E_{\mathrm{c}}}{\left(1+\left(E(x) / E_{\mathrm{c}}\right)^{\beta}\right)^{1 / \beta}}\right)
\end{array}\right.
$$

where $v$ stands for velocity, $\mu$ for mobility, $E$ for intensity, $V_{\text {dep }}$ for depletion voltage, $V$ for bias voltage and $d$ for detector thickness, one gets the carrier drift time $t_{\text {drift. }}$ The time is used to estimate 
the effect of diffusion, where diffusivity $D$ is calculated using the Einstein relation (4.2):

$$
D=\frac{k T}{q} \bar{\mu} \quad \longrightarrow \quad \sigma=\sqrt{2 D t_{\mathrm{drift}}}
$$

Here, $k$ represents the Boltzman constant, $T$ temperature, $q$ carrier charge and $\bar{\mu}$ average mobility. As a fast numerical technique the Romberg integration method has been used. The algorithm exploits a very general idea of Richardson's deferred approach to the limit, where a function is first integrated using trapezium rule and then the result is extrapolated to the limit (see [7]). After the carrier bunches have migrated to the electrode surface, they are diffused by multiple collisions and final Gaussian distributions (with $\sigma$ defined by (4.2)) are mapped to the strip layout. In the presence of a magnetic field $B$, the centers of such distributions are first shifted by Lorentz angle (4.3). The right panel of Fig. 7 illustrates the dependence of Lorentz angle on $V_{\text {bias }}$ and carrier position $x$.

$$
\tan \left(\vartheta_{\mathrm{L}}\right)=\frac{\int_{x}^{d} \mu(E(x)) r B d x}{\int_{x}^{d} d x} \begin{cases}r=1.13+0.0008(273-T) & \text { for } \mathrm{e} \\ r=0.72-0.0005(273-T) & \text { for } \mathrm{h}\end{cases}
$$
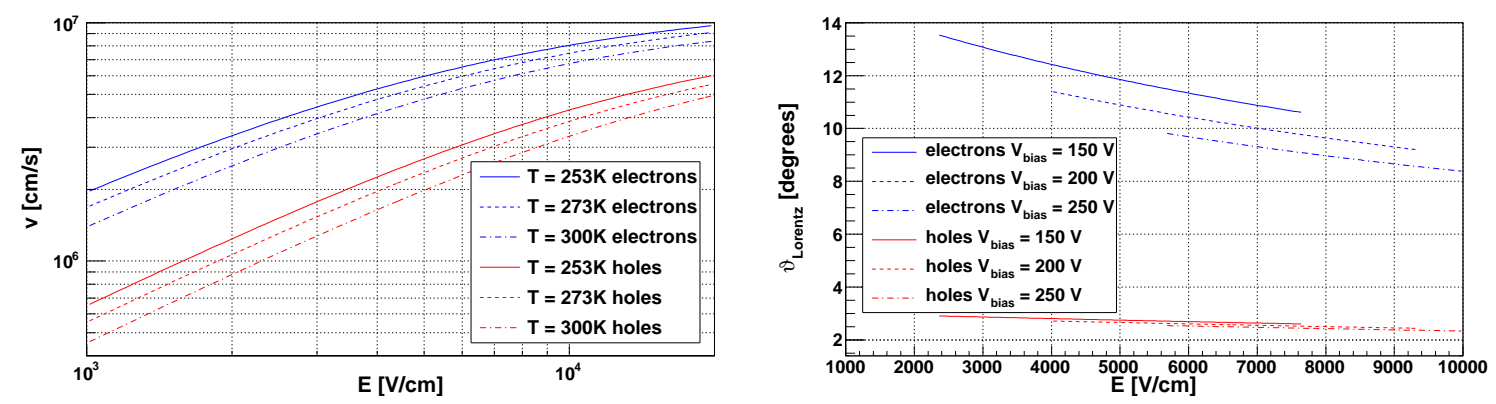

Figure 7: Left: Dependence of drift velocity versus intensity in a $300 \mu \mathrm{m}$ thick sensor for electrons (blue) and holes (red), both for different temperature: 253 (solid), 273 (dashed) and $300 \mathrm{~K}$ (semi-dashed). Right: Lorentz angle in $1.5 \mathrm{~T}$ magnetic field versus intensity (corresponding to $x$ position) for electrons (blue) and holes (red), both for different bias voltages: 150 (solid), 200 (dashed) and $250 \mathrm{~V}$ (semi-dashed), T=273 K.

Several electronic effects influence the final distribution of the collected charge on each strip. First, if the geometrical pitch is different from the read-out pitch, half of the charge is naturally collected by the left strip and the other half by the right strip. Furthermore, interstrip capacitance $C_{\mathrm{i}}$, strip-to-backside capacitance $C_{\mathrm{b}}$ and capacitive coupling $C_{\mathrm{c}}$, in case of AC coupled front-end electronics, are natural sources of mutual micro-strip crosstalk (charge sharing). Due to this effect, the charge collected on each read-out strip is redistributed again to neighbouring read-out strips as follows:

$$
i_{\text {neighbour }}=\frac{i_{\text {strip }} C_{\mathrm{i}}}{C_{\mathrm{b}}+C_{\mathrm{c}}+C_{\mathrm{i}}}
$$

An important capacitance here is the coupling capacitance, as it effectively enables to avoid the DC load by the continuously generated leakage current.

Second, common mode subtracted noise (CMS) is generated (using Gaussian distribution) and added to the signal. The typical sigma $\sigma_{\mathrm{cms}}$ of such a distribution usually depends on the length 
(capacitance) of the sensor and might be from several hundreds to thousands of e. Finally, if the read-out is binary, the charge above a specified threshold is set to 1 , and to 0 (discarded) otherwise. In case of an analog read-out, strips with a seed charge are found $\left(\sim 5 \sigma_{\text {cms }}\right.$ cut $)$ and then the neighbouring strips with charge above a given threshold $\left(\sim 2 \sigma_{\mathrm{cms}}\right)$ are added to the cluster.

After clustering, the double-sided or single-sided back-to-back detectors provide 2D spatial information, while single-sided can provide 1D information only. In case of shallow tracks, the number of hit strips, i.e. cluster size, might be higher than 3 and the center-of-gravity algorithm is not optimal anymore. Thus, the head-tail algorithm has been used, where $q_{\mathrm{L}}$ and $q_{\mathrm{R}}$ are the charges in the left- and rightmost strips and $\bar{q}_{\text {Inbtw }}$ is the average charge of intermediate strips [8].

$$
x_{\mathrm{COG}}=\frac{\sum_{\text {cluster }} x_{i} q_{i}}{\sum_{\text {cluster }} q_{i}} \quad x_{\text {HeadTail }}=\frac{x_{\mathrm{R}}+x_{\mathrm{L}}}{2}+\frac{q_{\mathrm{R}}-q_{\mathrm{L}}}{2 \bar{q}_{\text {Inbtw }}} \text { pitch }
$$

In case of double-sided sensors, the position is calculated independently in both directions and then combined into a TrackerHit. This gives rise to artificial hits (ghosts). Together with a 1D or a 2D spatial position a covariance matrix is estimated. The procedure consists in generating reasonable statistics of muons passing individual detectors at several $\vartheta$ angles $\left(20^{\circ}, 30^{\circ}, \ldots\right)$ and calculating the residuals (using MC truth information). The sigma of the gaussian, fitting the central $90 \%$ area of the residual distribution, defines then the TrackerHit resolution in the respective direction.

\subsection{SiPxlDigi - Physics Model}

Unlike a strip detector, the DEPleted Field Effect Transistor (DEPFET, [9]), represents a complex 2D device and a substantial simplification of in-detector physics processes is crucial for the digitization to perform in reasonable time. DEPFET can be described as a MOS-type field effect transistor integrated on a sidewards depleted $p$-on- $n$ silicon detector (Fig. 8). The device combines the advantages of fully depleted silicon sensor with in-pixel amplification. By means of sidewards depletion an additional deep $n$-implantation (a potential minimum for electrons) is created right underneath the transistor channel (see right Fig. 8). This can be regarded as an internal gate and when a particle creates e-h pairs, holes drift to the back contact and electrons are collected in the internal gate, where they accumulate. The signal charge then leads to a change in the potential of the internal gate, resulting in a modulation of the channel current of the transistor. After read-out, the signal charge is cleared out by a positive voltage pulse at the clear contact.
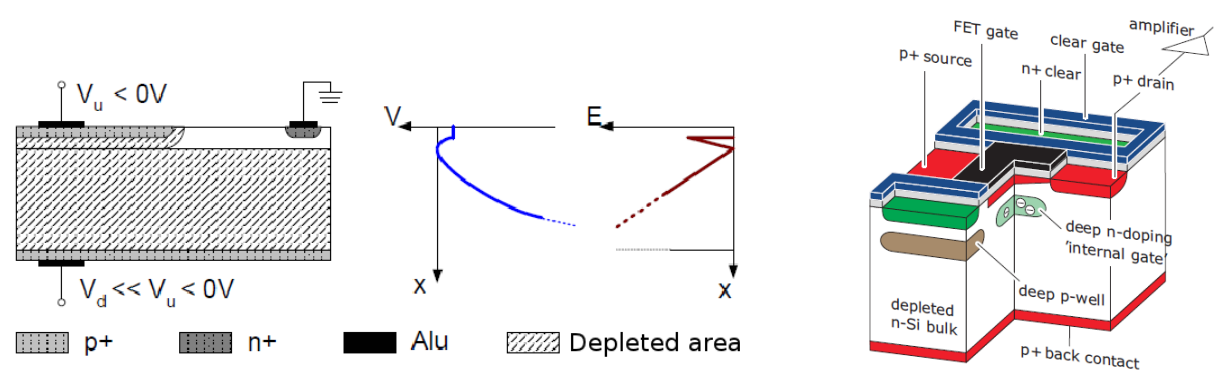

Figure 8: Left and Middle: Cross section of a sidewards depleted $p$-on- $n$ silicon detector and corresponding distribution of electric potential. Right: 2D scheme of DEPFET pixel. 
The generation of e-h pairs and continuous energy loss fluctuation is simulated in the same way as in SiStripDigi. What differs significantly is drift and diffusion (see Fig. 9). Based on full 3D device simulation ${ }^{1}$, we have divided a pixel matrix (see right Fig. 9) into drift and non-drift regions, which are defined as regions with and without lateral field ( $y$-axis) respectively. In drift
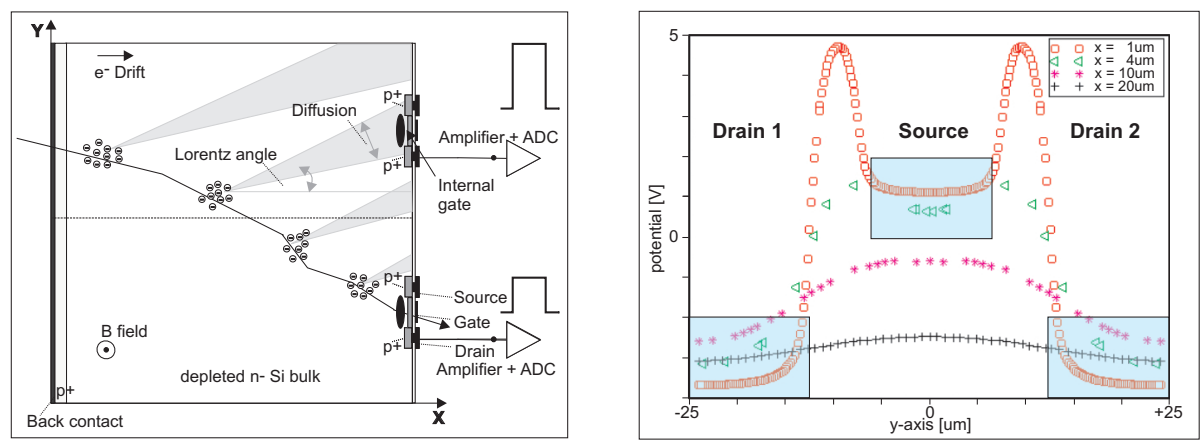

Figure 9: Left: A scheme of digitization procedure in DEPFET. Right: In-pixel potential distribution in $y$ direction for double-pixel structure and for different distances $x=1,4,10,20 \mu \mathrm{m}$ from the bottom of the FET structure. Blue regions with no lateral field are emphasized.

regions, longitudinal drift ( $x$-axis) of electrons in 1D parabolic shaped potential:

$$
\phi(x)=\frac{q N_{D}}{2 \varepsilon} x(d-x)+\frac{x}{d}\left(V_{\mathrm{x}=\mathrm{d}}-V_{\mathrm{x}=0}\right)+V_{\mathrm{x}=0} \quad \text { for } \quad N_{\mathrm{D}} \ll N_{\mathrm{A}}
$$

is performed up to a distance $\sim 10 \mu \mathrm{m}$ below surface. From there, electrons achieve potential minimum by lateral drift. The total drift time determines, as in SiStripDigi, a diffusion sigma and thus influences the diffusion spread. In non-drift regions, electrons achieve the minimum by mere diffusion (simulated as a random walk), which significantly increases the drift time and the corresponding cluster size. In the presence of magnetic field, the charge distribution is first shifted by the Lorentz angle (the angle is given as an input parameter) and the final charge distribution is then mapped to the pixel layout, so no FET effect is directly simulated. In the above relation: $q$ stands for carrier charge, $\varepsilon$ for permitivity, $d$ for detector thickness and $N_{\mathrm{D}}$ for the dopants concentration.

As DEPFET has almost no capacitive coupling between individual pixels, no charge-sharing effect is assumed. But there are other electronics effects, which are taken into account. A commonmode subtracted noise is added to the generated signal and an effect of ADC (analog-to-digital converter) is considered. In the ADC, the analog data are compared to digital thresholds (for a given number of bits: 5, 6,7 and 8) and the total charge is then translated into digits. After digitization, clustering is performed and COG or head-tail algorithms are used.

\subsection{Results and Models Validation}

The physics model implemented in the SiStripDigi package has been validated against the ATLAS SCT test beam data. For the validation, a simulation of $180 \mathrm{GeV} / \mathrm{c}$ pions passing a binary read-out micro-strip detector has been performed. Concerning the simulation and test beam conditions, the binary threshold has been set to $1 \mathrm{fC}$, detector noise to $\sigma_{\mathrm{cms}} \approx 1500 \mathrm{e}$ and telescope spatial

\footnotetext{
${ }^{1}$ performed by R. Richter, MPI Munich
} 

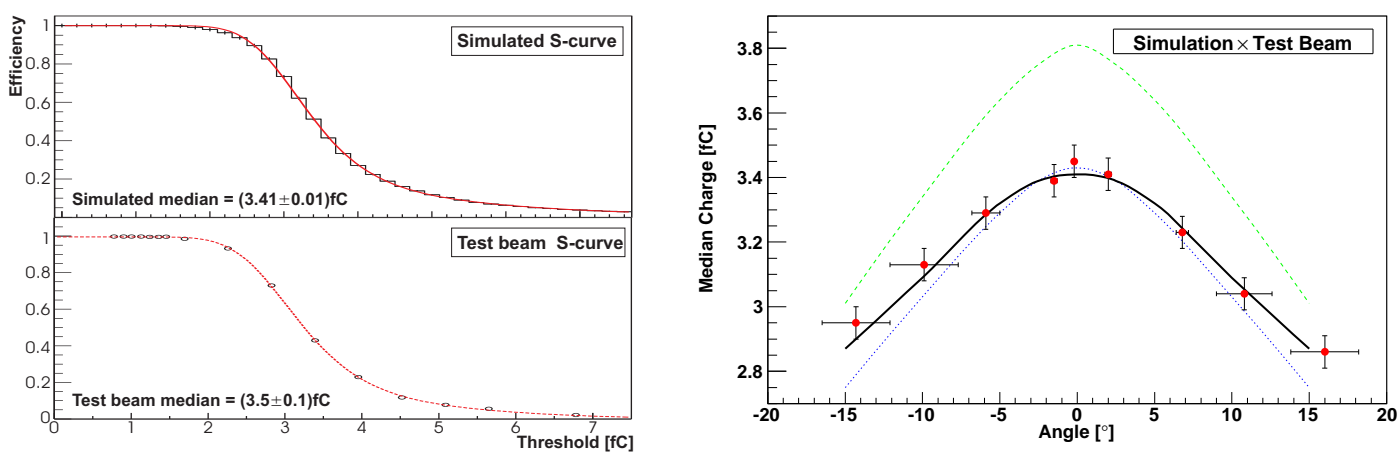

Figure 10: Left: Typical efficiency versus threshold for binary read-out detectors (S-curve): simulated median $=(3.41 \pm 0.01) \mathrm{fC}$ (top), experimental median $=(3.5 \pm 0.1) \mathrm{fC}$ (bottom). Right: Median collected charge versus incidence angle: simulations with drift and diffusion, no $\delta$-electrons simulated (green), drift, diffusion and cross-talk effects (blue), and full simulation with $\delta$-electrons and all digitization effects (black). Measurement results (test beam) are shown as red markers.

resolution to $\approx 5 \mu \mathrm{m}$. See [10] and [3] for details. To determine the importance of individual effects, the simulation has been performed with and without $\delta$-electrons (difference between black and blue curve in Fig. 10), and with and without mutual cross-talk effect (difference between green and blue curve). The simulated S-curve (binary threshold scan) and the corresponding median, as well as the median charge versus incidence angle, perfectly agree with the measured data.
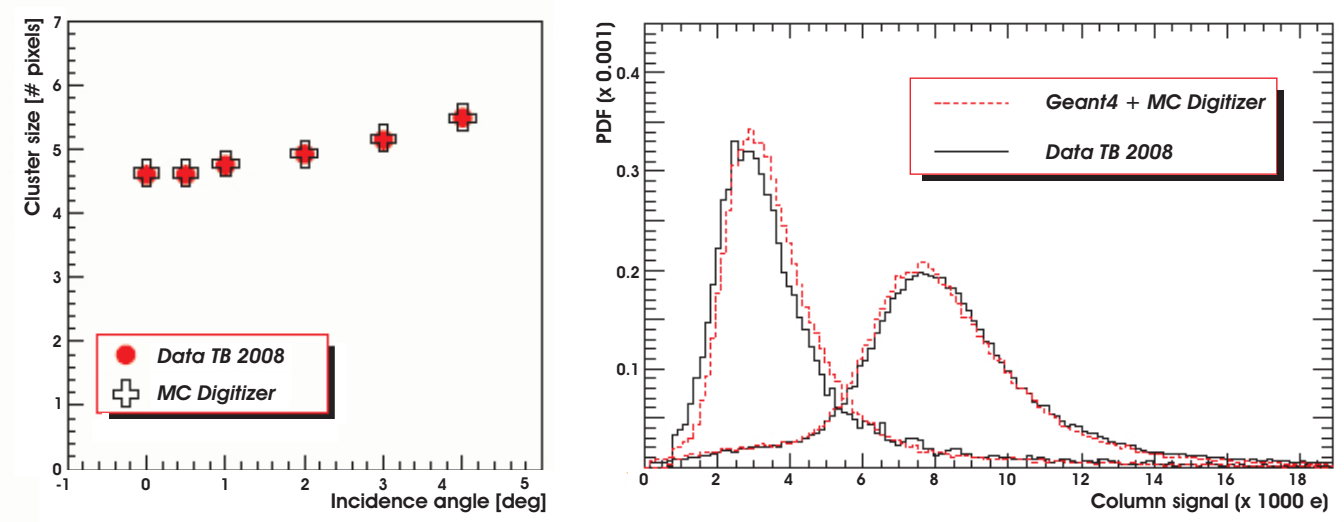

Figure 11: Left: Cluster size versus incidence angle. Right: Single-column signal distribution for inclined tracks. Right peak corresponds to passage through $450 \mu \mathrm{m}$ thick silicon (no inclined tracks), left peak corresponds to effective passage through $50 \mu \mathrm{m}$ thick silicon (inclined tracks) - signal extraction from the thick detector in order to estimate signal generation in thinned detectors.

The physics model implemented in SiPxIDigi has been validated against DEPFET test beam data [11]. For the validation a simulation of $120 \mathrm{GeV} / \mathrm{c}$ pions in a $450 \mu \mathrm{m}$ thick DEPFET detector has been performed and particular focus has been given on correct explanation of cluster size measurements and signal charge generation, namely for inclined tracks. Both distributions are depicted in Fig. 11. Particularly interesting is the signal generation for inclined incident particles, 
where the charge deposited in each pixel cell might be roughly the same as in future thinned down Belle II-like DEPFET detectors.

\section{Summary}

We have described our approach to the simulation of pixel detectors (DEPFETs) and silicon micro-strip detectors for the Belle II experiment using the ILC software framework. Furthermore, we have addressed possible issues connected with such simulation in Geant 4 and explained in detail our physics models, which are used to describe in-detector physics processes to simulate the response of such devices. And finally, we have demonstrated the validity of the models against the test beam data.

\section{References}

[1] Belle II collaboration, Belle II Technical Design Report, arXiv: 1011.0352, KEK 2010

[2] ILC collaboration, [http://ilcsoft.desy.de/portal]

[3] Z. Drasal, Simulation of Charge Collection in Semiconductor Microstrip Detectors, Charles University, Prague 2006, [http: //www-ucjf.troja.mff.cuni.cz/diploma.php]

[4] Geant4 collaboration, Physics Reference Manual, CERN, Geneve 2010, [http://geant 4.cern.ch/support/userdocuments.shtml]

[5] H. Bichsel, Straggling in Thin Silicon Detectors, Reviews of Modern Physics 60 (663-699)

[6] M. Brigida et al., A new Monte Carlo code for full simulation of silicon strip detectors, NIMA $\mathbf{5 3 3}$ (322-343)

[7] J.Stoer, R.Bulirsch, Introduction to Numerical Analysis, 3rd edition Springer, New York 2002

[8] R. Turchetta, Spatial resolution of silicon microstrip detectors, NIMA 335 (44-58)

[9] J. Kemmer, G. Lutz, New semiconductor detector concepts, NIMA 253 (356)

[10] F. Campabadal et al., Beam Tests of ATLAS SCT Silicon Strip Detector Modules, NIMA 538 (384-407)

[11] L. Andricek et al., Intrinsic resolutions of DEPFET detector prototypes measured at beam tests, NIMA $638(24-32)$ 